\title{
Poisson-Lie T-plurality revisited. Is T-duality unique?
}

\author{
Ladislav Hlavatý $\dot{y}^{a}$ and Ivo Petr ${ }^{b}$ \\ ${ }^{a}$ Department of Physics, Faculty of Nuclear Sciences and Physical Engineering, \\ Czech Technical University in Prague, Břehová 7, Prague 1, 11519 Czech Republic \\ ${ }^{b}$ Department of Applied Mathematics, Faculty of Information Technology, \\ Czech Technical University in Prague, Thákurova 9, Prague 6, 16000 Czech Republic \\ E-mail: hlavaty@fjfi.cvut.cz, ivo.petr@fit.cvut.cz
}

Abstract: We investigate (non-)Abelian T-duality from the perspective of Poisson-Lie T-plurality. We show that sigma models related by duality/plurality are given not only by Manin triples obtained from decompositions of Drinfel'd double, but also by their particular embeddings, i.e. maps that relate bases of these decompositions. This allows us to get richer set of dual or plural sigma models than previously thought. That's why we ask how Tduality is defined and what should be the "canonical" duality or plurality transformation.

Keywords: Sigma Models, String Duality, Integrable Field Theories

ArXIV EPRINT: 1811.12235 


\section{Contents}

1 Introduction 1

2 Poisson-Lie T-plurality of sigma models 2

2.1 Poisson-Lie T-plurality with spectators 3

2.2 Equivalence of transformation matrices 5

3 Sigma models with two-dimensional target space $\quad 5$

$\begin{array}{lll}3.1 & \text { Poisson-Lie T-plurality } & 6\end{array}$

3.2 Poisson-Lie T-duality 8

4 Sigma models with four-dimensional target space 10

5 Poisson-Lie T-dualities and pluralities generated by Poisson-Lie iden$\begin{array}{ll}\text { tities } & 12\end{array}$

6 Conclusion

\section{Introduction}

The notion of (non-)Abelian T-duality [1-3] of sigma models relies on the presence of symmetries of the sigma model backgrounds. Whenever there is such a symmetry, one may gauge it to arrive at a model related to the original one by T-duality. This technique, extended to RR fields in $[4,5]$, is used frequently to generate new supergravity solutions, see e.g. $[6,7]$ and references therein. Non-Abelian T-duality, however, does not preserve the symmetries, and it may not be possible to return back to the initial model. Poisson-Lie T-duality, introduced in the seminal paper [8] by Klimčík and Ševera, treats both models equally and offers a remedy to this issue.

The algebraic structure underlying Poisson-Lie T-duality is the Drinfel'd double, a Lie group $\mathscr{D}$ that decomposes into two Lie subgroups $\mathscr{G}$ and $\widetilde{\mathscr{G}}$ of equal dimension. In case of (non-)Abelian T-duality the former represents group of symmetries of the initial sigma model, while the latter is Abelian. There are also Drinfel'd doubles where both $\mathscr{G}$ and $\widetilde{\mathscr{G}}$ are non-Abelian. In such a case the symmetry of the initial model is replaced by the so-called Poisson-Lie symmetry (or generalized symmetry), see [9], and the full Poisson-Lie T-duality transformation applies. Nevertheless, the presence of symmetries remains crucial if one wants to dualize a particular background [10]. Recently (PoissonLie) T-duality also appears as an important tool in the study of integrable models and their deformations [11-13]. 
Since duality exchanges roles of $\mathscr{G}$ and $\widetilde{\mathscr{G}}$, we may understand it in terms of Drinfel'd double as a switch between decompositions $(\mathscr{G} \mid \widetilde{\mathscr{G}})$ and $(\widetilde{\mathscr{G}} \mid \mathscr{G})$ of $\mathscr{D}$. The authors of [8] mention the fact that a Drinfel'd double $\mathscr{D}$ can have other decompositions $(\mathcal{K} \mid \widetilde{\mathcal{K}}),(\widetilde{\mathcal{K}} \mid \mathcal{K}), \ldots$ beside $(\mathscr{G} \mid \widetilde{\mathscr{G}})$ and $(\widetilde{\mathscr{G}} \mid \mathscr{G})$. All these decompositions can be used to construct mutually related sigma models. The transformation of the initial model constructed by decomposition $(\mathscr{G} \mid \widetilde{\mathscr{G}})$ to a model constructed by $(\mathcal{K} \mid \widetilde{\mathcal{K}})$ was later denoted Poisson-Lie T-plurality [14]. Examples of sigma models related by Poisson-Lie T-plurality were studied e.g. in [14, 15], and decompositions of low-dimensional Drinfel'd doubles were classified in [16-18].

The goal of this paper is to show, using simple examples, that sigma models related by Poisson-Lie T-duality/plurality are given not only by the algebraic structure of decompositions of Lie algebra of the Drinfel'd double into Manin triples, but also by the particular embedding of Manin triples, i.e. maps that relate bases in various decompositions. For this purpose we shall consider the simplest possible case of Drinfel'd double accomodating plurality, i.e. a four-dimensional semi-Abelian Drinfel'd doubles.

After summarizing Poisson-Lie T-plurality in section 2 we identify transformations that yield equivalent sigma model backgrounds. In section 3 we develop examples of dual/plural models whose geometric properties depend on the choice of matrices transforming bases of Manin triples, and, in section 4, we show that nonequivalent models can be obtained even if we do not change the Manin triple at all. We study this "Poisson-Lie T-identity" further in section 5 trying to identify what the "canonical" duality/plurality should be.

\section{Poisson-Lie T-plurality of sigma models}

Let $\mathscr{M}$ be $n$-dimensional (pseudo-)Riemannian target manifold and consider sigma model on $\mathscr{M}$ given by Lagrangian

$$
\mathcal{L}=\partial_{-} \phi^{\mu} \mathcal{F}_{\mu \nu}(\phi) \partial_{+} \phi^{\nu}, \quad \phi^{\mu}=\phi^{\mu}\left(x_{+}, x_{-}\right), \quad \mu=1, \ldots, n
$$

where tensor $\mathcal{F}=\mathcal{G}+\mathcal{B}$ defines metric and torsion potential of the target manifold. Assume that there is a $d$-dimensional Lie group $\mathscr{G}$ with free action on $\mathscr{M}$ that leaves the tensor invariant. The action of $\mathscr{G}$ is transitive on its orbits, hence we may locally consider $\mathscr{M} \approx(\mathscr{M} / \mathscr{G}) \times \mathscr{G}=\mathscr{N} \times \mathscr{G}$, and introduce adapted coordinates

$$
x^{\mu}=\left\{s_{\delta}, g_{a}\right\}, \quad \delta=1, \ldots, n-d, \quad a=1, \ldots, d
$$

where $s_{\delta}$ label the orbits of $\mathscr{G}$ and are treated as spectators and $g_{a}$ are group coordinates [19, 20]. Dualizable sigma model on $\mathscr{N} \times \mathscr{G}$ is given by tensor field $\mathcal{F}$ defined by $n \times n$ matrix $E(s)$ as

$$
\mathcal{F}(s, g)=\mathcal{E}(g) \cdot E(s) \cdot \mathcal{E}^{T}(g), \quad \mathcal{E}(g)=\left(\begin{array}{cc}
1 & 0 \\
0 & e(g)
\end{array}\right)
$$

where $e(g)$ is $d \times d$ matrix of components of right-invariant Maurer-Cartan form $(d g) g^{-1}$ on $\mathscr{G}$. 
Using non-Abelian T-duality one can find dual sigma model on $\mathscr{N} \times \widetilde{\mathscr{G}}$, where $\widetilde{\mathscr{G}}$ is Abelian subgroup of semi-Abelian Drinfel'd double $\mathscr{D}$ that splits into subgroups $\mathscr{G}$ and $\widetilde{\mathscr{G}}$. The necessary formulas will be given in the following subsection as a special case of PoissonLie T-plurality. In papers [21-23], non-Abelian T-duals of sigma model in flat torsionless four-dimensional background were constructed. The groups $\mathscr{G}$ were then subgroups of the Poincaré group [24].

\subsection{Poisson-Lie T-plurality with spectators}

For certain Drinfel'd doubles several decompositions may exist. Suppose that $\mathscr{D}=(\mathscr{G} \mid \widetilde{\mathscr{G}})$ splits into another pair of subgroups $\widehat{\mathscr{G}}$ and $\overline{\mathscr{G}}$. Then we can apply the full framework of Poisson-Lie T-plurality $[8,14]$ and find sigma model on $\mathscr{N} \times \widehat{\mathscr{G}}$.

The $2 d$-dimensional Lie algebra $\mathfrak{d}$ of the Drinfel'd double $\mathscr{D}$ is equipped with an adinvariant non-degenerate symmetric bilinear form $\langle.,$.$\rangle . Let \mathfrak{d}=\mathfrak{g} \oplus \tilde{\mathfrak{g}}$ and $\mathfrak{d}=\widehat{\mathfrak{g}} \oplus \overline{\mathfrak{g}}$ be two decompositions (Manin triples $(\mathfrak{d}, \mathfrak{g}, \tilde{\mathfrak{g}})$ and $(\mathfrak{d}, \widehat{\mathfrak{g}}, \overline{\mathfrak{g}}))$ of $\mathfrak{d}$ into subalgebras that are maximally isotropic with respect to $\langle.,$.$\rangle . The pairs of mutually dual bases T_{a} \in \mathfrak{g}, \widetilde{T}^{a} \in \tilde{\mathfrak{g}}$ and $\widehat{T}_{a} \in \widehat{\mathfrak{g}}, \bar{T}^{a} \in \overline{\mathfrak{g}}, a=1, \ldots, d$, satisfying

$$
\begin{array}{lll}
\left\langle T_{a}, T_{b}\right\rangle=0, & \left\langle\widetilde{T}^{a}, \widetilde{T}^{b}\right\rangle=0, & \left\langle T_{a}, \widetilde{T}^{b}\right\rangle=\delta_{a}^{b}, \\
\left\langle\widehat{T}_{a}, \widehat{T}_{b}\right\rangle=0, & \left\langle\bar{T}^{a}, \bar{T}^{b}\right\rangle=0, & \left\langle\widehat{T}_{a}, \bar{T}^{b}\right\rangle=\delta_{a}^{b}
\end{array}
$$

are related by transformation

$$
\left(\begin{array}{l}
\widehat{T} \\
\bar{T}
\end{array}\right)=C \cdot\left(\begin{array}{c}
T \\
\widetilde{T}
\end{array}\right)
$$

where $C$ is an invertible $2 d \times 2 d$ matrix. Due to ad-invariance of the bilinear form $\langle.,$.$\rangle the$ algebraic structure of $\mathfrak{d}$ is given both by

$$
\left[T_{i}, T_{j}\right]=f_{i j}^{k} T_{k}, \quad\left[\widetilde{T}^{i}, \widetilde{T}^{j}\right]=\tilde{f}_{k}^{i j} \widetilde{T}^{k}, \quad\left[T_{i}, \widetilde{T}^{j}\right]=f_{k i}^{j} \widetilde{T}^{k}+\tilde{f}_{i}^{j k} T_{k}
$$

and

$$
\left[\widehat{T}_{i}, \widehat{T}_{j}\right]=\hat{f}_{i j}^{k} \widehat{T}_{k}, \quad\left[\bar{T}^{i}, \bar{T}^{j}\right]=\bar{f}_{k}^{i j} \bar{T}^{k}, \quad\left[\widehat{T}_{i}, \bar{T}^{j}\right]=\hat{f}_{k i}^{j} \bar{T}^{k}+\bar{f}_{i}^{j k} \widehat{T}_{k} .
$$

Given the structure constants $F_{i j}^{k}$ of $\mathfrak{d}=\mathfrak{g} \oplus \tilde{\mathfrak{g}}$ and $\widehat{F}_{i j}^{k}$ of $\mathfrak{d}=\widehat{\mathfrak{g}} \oplus \overline{\mathfrak{g}}$, the matrix $C$ has to satisfy equation ${ }^{1}$

$$
C_{a}^{p} C_{b}^{q} F_{p q}^{r}=\widehat{F}_{a b}^{c} C_{c}^{r}
$$

To preserve the bilinear form $\langle.,$.$\rangle and thus (2.4), C$ also has to satisfy

$$
C_{a}^{p} C_{b}^{q} B_{p q}=B_{a b}
$$

where $B_{a b}$ are components of matrix $B$ that can be written in block form as

$$
B=\left(\begin{array}{ll}
\mathbf{0}_{d} & \mathbf{1}_{d} \\
\mathbf{1}_{d} & \mathbf{0}_{d}
\end{array}\right) .
$$

\footnotetext{
${ }^{1}$ Conditions on $C$ 's are more restrictive than those for "NATD group" investigated in [25] (we are grateful to D. Osten for bringing our attention to this paper) but as said in the Introduction, our main goal is to present dependence of geometrical properties of the Poisson-Lie plural sigma models on matrices $C$.
} 
In other words, $C$ is an element of $O(d, d)$ but, unlike the case of Abelian T-duality, not every element of $O(d, d)$ is allowed in (2.5).

For the following formulas it will be convenient to introduce $d \times d$ matrices $P, Q, R, S$ as

$$
\left(\begin{array}{l}
T \\
\widetilde{T}
\end{array}\right)=C^{-1} \cdot\left(\begin{array}{l}
\widehat{T} \\
\bar{T}
\end{array}\right)=\left(\begin{array}{ll}
P & Q \\
R & S
\end{array}\right) \cdot\left(\begin{array}{l}
\widehat{T} \\
\bar{T}
\end{array}\right) .
$$

To accommodate the spectator fields we have to extend these to $n \times n$ matrices

$$
\mathcal{P}=\left(\begin{array}{ll}
1 & 0 \\
0 & P
\end{array}\right), \quad \mathcal{Q}=\left(\begin{array}{ll}
0 & 0 \\
0 & Q
\end{array}\right), \quad \mathcal{R}=\left(\begin{array}{ll}
0 & 0 \\
0 & R
\end{array}\right), \quad \mathcal{S}=\left(\begin{array}{ll}
1 & 0 \\
0 & S
\end{array}\right) .
$$

It is also advantageous to introduce block form of $E(s)$ as

$$
E(s)=\left(\begin{array}{cc}
E_{\alpha \beta}(s) & E_{\alpha b}(s) \\
E_{a \beta}(s) & E_{a b}(s)
\end{array}\right), \quad \alpha, \beta=1, \ldots, n-d, \quad a, b=1, \ldots, d
$$

The sigma model on $\mathscr{N} \times \widehat{\mathscr{G}}$ related to (2.3) via Poisson-Lie T-plurality is given by tensor $\widehat{\mathcal{F}}(s, \hat{g})$ that is calculated as

$$
\widehat{\mathcal{F}}(s, \hat{g})=\widehat{\mathcal{E}}(\hat{g}) \cdot \widehat{E}(s, \hat{g}) \cdot \widehat{\mathcal{E}}^{T}(\hat{g}), \quad \widehat{\mathcal{E}}(\hat{g})=\left(\begin{array}{cc}
1 & 0 \\
0 & \widehat{e}(\hat{g})
\end{array}\right)
$$

where

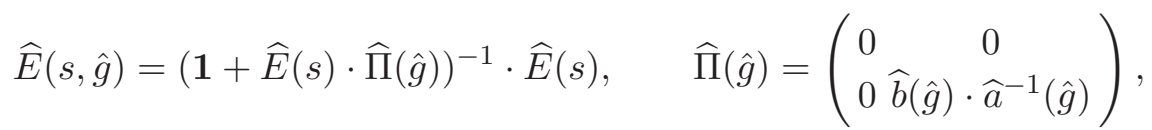

matrices $\widehat{b}(\hat{g})$ and $\widehat{a}(\hat{g})$ are submatrices of the adjoint representation

$$
a d_{\hat{g}^{-1}}(\widehat{T})=\widehat{b}(\hat{g}) \cdot \bar{T}+\widehat{a}^{-1}(\hat{g}) \cdot \widehat{T},
$$

and the matrix $\widehat{E}(s)$ is obtained by formula

$$
\widehat{E}(s)=(\mathcal{P}+E(s) \cdot \mathcal{R})^{-1} \cdot(\mathcal{Q}+E(s) \cdot \mathcal{S}) .
$$

Therefore, it is necessary that

$$
\operatorname{det}(\mathcal{P}+E(s) \cdot \mathcal{R})=\operatorname{det}\left(P+E_{a b}(s) \cdot R\right) \neq 0 .
$$

These formulas reduce to formulas for Poisson-Lie T-duality if we choose $P=S=\mathbf{0}_{d}$ and $Q=R=\mathbf{1}_{d}$. Furthermore, for a semi-Abelian Drinfel'd double the well-known Buscher rules for non-Abelian T-duality are restored. If there are no spectators, i.e. if $n=d$, the plurality is called atomic. 


\subsection{Equivalence of transformation matrices}

Tensors $\mathcal{F}, \widehat{\mathcal{F}}$ are expressed by formulas (2.3) and (2.9) in particular bases of subalgebras $\mathfrak{g}, \widehat{\mathfrak{g}}$ of Manin triples $(\mathfrak{d}, \mathfrak{g}, \tilde{\mathfrak{g}})$ and $(\mathfrak{d}, \widehat{\mathfrak{g}}, \overline{\mathfrak{g}})$. However, both initial and dual/plural tensor do not depend on the choice of bases in $\mathfrak{g}$ or $\widehat{\mathfrak{g}}$. Their geometric properties are thus independent as well.

Let us consider automorphisms of both Manin triples given by linear transformations of $\mathfrak{g}$ and $\widehat{\mathfrak{g}}$ that preserve their algebraic structure. Let $A$ and $B$ be $d \times d$ matrices that transform bases $T_{a}$ and $\widehat{T}_{a}$. Transformations $(2.5)$ of the form

$$
\left(\begin{array}{c}
T^{\prime} \\
\widetilde{T}^{\prime}
\end{array}\right)=\left(\begin{array}{cc}
A & 0 \\
0 & A^{-T}
\end{array}\right) \cdot\left(\begin{array}{c}
T \\
\widetilde{T}
\end{array}\right), \quad\left(\begin{array}{c}
\widehat{T}^{\prime} \\
\bar{T}^{\prime}
\end{array}\right)=\left(\begin{array}{cc}
B & 0 \\
0 & B^{-T}
\end{array}\right) \cdot\left(\begin{array}{c}
\widehat{T} \\
\bar{T}
\end{array}\right)
$$

then preserve the algebraic structure $(2.6),(2.7)$ and duality $(2.4)$ of bases $(T, \widetilde{T})$ and $(\widehat{T}, \bar{T})$.

Transformations (2.14) induce changes in matrices $E(s)$ and $\widehat{E}(s)$ that are used in construction of background tensors. We have

$$
E^{\prime}(s)=\mathcal{A} \cdot E(s) \cdot \mathcal{A}^{T}, \quad \widehat{E}^{\prime}(s)=\mathcal{B} \cdot \widehat{E}(s) \cdot \mathcal{B}^{T}
$$

where

$$
\mathcal{A}=\left(\begin{array}{ll}
1 & 0 \\
0 & A
\end{array}\right), \quad \mathcal{B}=\left(\begin{array}{ll}
1 & 0 \\
0 & B
\end{array}\right) .
$$

If the relation of bases in Manin triples is written as in (2.8), then it is easy to check that

$$
\left(\begin{array}{l}
T^{\prime} \\
\widetilde{T}^{\prime}
\end{array}\right)=\left(\begin{array}{ll}
P^{\prime} & Q^{\prime} \\
R^{\prime} & S^{\prime}
\end{array}\right) \cdot\left(\begin{array}{c}
\widehat{T}^{\prime} \\
\bar{T}^{\prime}
\end{array}\right)
$$

where

$$
\left(\begin{array}{ll}
P^{\prime} & Q^{\prime} \\
R^{\prime} & S^{\prime}
\end{array}\right)=\left(\begin{array}{cc}
A P B^{-1} & A Q B^{T} \\
A^{-T} R B^{-1} & A^{-T} S B^{T}
\end{array}\right)
$$

Therefore, matrices

$$
\left(\begin{array}{ll}
P & Q \\
R & S
\end{array}\right) \text { and }\left(\begin{array}{ll}
P^{\prime} & Q^{\prime} \\
R^{\prime} & S^{\prime}
\end{array}\right)
$$

define transformations between sigma model backgrounds $\mathcal{F}$ and $\widehat{\mathcal{F}}$ in various coordinates. That's why, from the perspective of Poisson-Lie T-plurality, they can be considered equivalent.

\section{Sigma models with two-dimensional target space}

In this section we shall consider atomic Poisson-Lie T-plurality of sigma models whose target space is a two-dimensional solvable Lie group $\mathscr{G}$ with generators $T_{1}, T_{2}$ satisfying

$$
\left[T_{1}, T_{2}\right]=T_{2} .
$$


The trace $f_{i k}^{k}$ of the structure constants is not zero since $f_{12}^{2}=1$ and it is known that this leads to mixed gauge and gravitational anomaly [26] in the dual model. Yet, it is worth considering such groups in the context of integrable models [27] and generalized supergravity [28]. We parametrize the elements $g \in \mathscr{G}$ as $g=\mathrm{e}^{g_{1} T_{1}} \mathrm{e}^{g_{2} T_{2}}$. Since there are no spectators, the matrix $E(s)$ is constant. Choosing it in the form

$$
E(s)=\left(\begin{array}{cc}
\alpha & \beta \\
\gamma & 0
\end{array}\right)
$$

we find that the background tensor $\mathcal{F}\left(g_{1}, g_{2}\right)$ calculated according to the formula (2.3) is given by

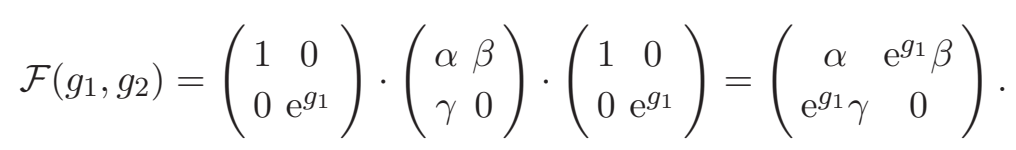

One can verify that $\mathcal{F}$ is invariant with respect to $\mathscr{G}$ and its symmetric part $\mathcal{G}$ is flat metric. Since the target manifold is two-dimensional, the torsion $H=d \mathcal{B}$ of all the backgrounds discussed in this section vanishes. Therefore, the $\mathcal{B}$-field can be eliminated by a gauge transformation and the only relevant part of $\mathcal{F}$ is the metric $\mathcal{G}$.

\subsection{Poisson-Lie T-plurality}

In order to find Poisson-Lie T-dual or plural models associated to (3.2), we embed $\mathscr{G}$ into four-dimensional Drinfel'd double $\mathscr{D}=(\mathscr{G} \mid \widetilde{\mathscr{G}})$ with $\widetilde{\mathscr{G}}$ Abelian. The algebraic structure of four-dimensional Drinfel'd doubles was studied in [17], where it was shown that for such Drinfel'd double there are two nonequivalent Manin triples:

- Semi-Abelian triple $\mathfrak{d}=\mathfrak{g} \oplus \tilde{\mathfrak{g}}$ with dual basis $\left(T_{1}, T_{2}, \widetilde{T}^{1}, \widetilde{T}^{2}\right)$ and Lie brackets (only nontrivial brackets are displayed)

$$
\left[T_{1}, T_{2}\right]=T_{2}, \quad\left[T_{1}, \widetilde{T}^{2}\right]=-\widetilde{T}^{2}, \quad\left[T_{2}, \widetilde{T}^{2}\right]=\widetilde{T}^{1},
$$

or

- Type B non-Abelian triple $\mathfrak{d}=\widehat{\mathfrak{g}} \oplus \overline{\mathfrak{g}}$ with dual basis $\left(\widehat{T}_{1}, \widehat{T}_{2}, \bar{T}^{1}, \bar{T}^{2}\right)$ and Lie brackets

$$
\begin{aligned}
{\left[\widehat{T}_{1}, \widehat{T}_{2}\right] } & =\widehat{T}_{2}, & {\left[\bar{T}^{1}, \bar{T}^{2}\right] } & =\bar{T}^{1}, \\
{\left[\widehat{T}_{1}, \bar{T}^{1}\right] } & =\widehat{T}_{2}, & {\left[\widehat{T}_{1}, \bar{T}^{2}\right] } & =-\widehat{T}_{1}-\bar{T}^{2},
\end{aligned}
$$

Map relating both bases

$$
\begin{array}{ll}
\widehat{T}_{1}=-T_{1}+T_{2}, & \widehat{T}_{2}=\widetilde{T}^{1}+\widetilde{T}^{2}, \\
\bar{T}^{1}=\widetilde{T}^{2}, & \bar{T}^{2}=T_{1}
\end{array}
$$


mentioned in the paper [17] preserves (2.4) and transforms Lie brackets (3.3) into (3.4). However, there are two different classes of linear maps (2.5) given by matrices

$$
C_{1}=\left(\begin{array}{cccc}
-1 & b_{2} & b_{2} b_{3} & b_{3} \\
0 & 0 & b_{1} b_{2} & b_{1} \\
0 & 0 & b_{1} b_{2}-1 & b_{1} \\
1 & \frac{1}{b_{1}}-b_{2} & \left(\frac{1}{b_{1}}-b_{2}\right) b_{3} & -b_{3}
\end{array}\right), \quad b_{1}, b_{2}, b_{3} \in \mathbb{R}, b_{1} \neq 0
$$

or

$$
C_{2}=\left(\begin{array}{cccc}
1 & b_{2} & -b_{2} b_{3} & b_{3} \\
0 & b_{1} & -b_{1} b_{3} & 0 \\
0 & b_{1} & 1-b_{1} b_{3} & 0 \\
-1 & -b_{2} & \frac{b_{2}\left(b_{1} b_{3}-1\right)}{b_{1}} & \frac{1}{b_{1}}-b_{3}
\end{array}\right), \quad b_{1}, b_{2}, b_{3} \in \mathbb{R}, b_{1} \neq 0
$$

that do the same and that define much richer set of decompositions. ${ }^{2}$ Note that $\operatorname{det} C_{1}=-1$ and $\operatorname{det} C_{2}=1$. We will show that using these two maps to generate models "plural" to $(2.1)$ with $\mathcal{F}$ given by (3.2) one gets substantially different models.

The first one, obtained from (2.9)-(2.12) and (3.6), is given by background ${ }^{3}$

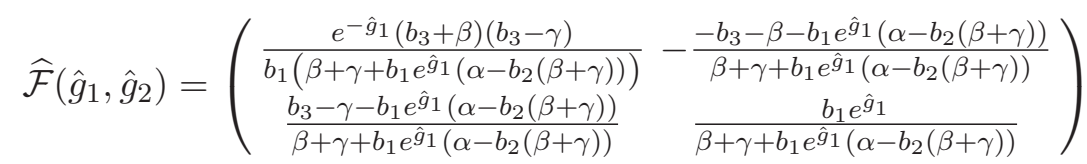

with nonzero $\left(b_{1} \neq 0\right)$ scalar curvature

$$
\widehat{R}=-\frac{4 b_{1} e^{\hat{g}_{1}}}{\beta+b_{1} e^{\hat{g}_{1}}\left(\alpha-b_{2}(\beta+\gamma)\right)+\gamma} .
$$

The other one, obtained using (3.7), is given by

$$
\widehat{\mathcal{F}}\left(\hat{g}_{1}, \hat{g}_{2}\right)=\left(\begin{array}{cc}
\frac{\alpha+b_{2}(\beta+\gamma)}{\left(b_{1} e^{\hat{g}_{1}}\left(b_{3}-\beta\right)-1\right)\left(b_{1} e^{\hat{g}_{1}}\left(b_{3}+\gamma\right)-1\right)} & \frac{b_{1} e^{\hat{g}_{1}}\left(b_{3}-\beta\right)}{b_{1} e^{\hat{g}_{1}}\left(b_{3}-\beta\right)-1} \\
-\frac{b_{1} e^{\hat{g}_{1}}\left(b_{3}+\gamma\right)}{b_{1} e^{\hat{g}_{1}}\left(b_{3}+\gamma\right)-1} & 0
\end{array}\right) .
$$

This is a background with flat metric, so indeed, using Poisson-Lie T-plurality with two different maps (3.6) and (3.7) we get two different sigma models. This essential difference of curvature properties of the backgrounds remains true for any choice of $b_{1}, b_{2}, b_{3}$.

Similar results are obtained if we consider plural sigma models on $\overline{\mathscr{G}}$. In that case we use transformations between bases of semi-Abelian Manin triple and a "dual" to type-B Manin triple, i.e. the matrices (3.6) and (3.7) are multiplied from the left by the exchange matrix

$$
D_{0}=\left(\begin{array}{llll}
0 & 0 & 1 & 0 \\
0 & 0 & 0 & 1 \\
1 & 0 & 0 & 0 \\
0 & 1 & 0 & 0
\end{array}\right)
$$

\footnotetext{
${ }^{2}$ One can simplify these matrices by $(2.14),(2.16)$ and choose e.g. $b_{1}=1, b_{2}=b_{3}=0$ for $C_{1}$ and $b_{2}=0$ for $C_{2}$. To get the map (3.5) one has to choose $b_{1}=b_{2}=1, b_{3}=0$.

${ }^{3}$ We assume that elements of $\widehat{\mathscr{G}}$ are parametrized as $\hat{g}=\mathrm{e}^{\hat{g}_{1} \widehat{T}_{1}} \mathrm{e}^{\hat{g}_{2} \widehat{T}_{2}}$.
} 


\subsection{Poisson-Lie T-duality}

Maybe surprisingly, we observe the same phenomenon for Poisson-Lie T-duality as well. Dual sigma models can be obtained by exchange of Manin triples $(\mathfrak{d}, \mathfrak{g}, \tilde{\mathfrak{g}})$ and $(\mathfrak{d}, \tilde{\mathfrak{g}}, \mathfrak{g})$ mediated by the matrix (3.9). On the other hand, there are more general maps between bases of the semi-Abelian Manin triple and its dual. Linear maps on $\mathfrak{d}$ that switch the roles of $T_{a}$ and $\widetilde{T}^{a}$ in (3.3) and meanwhile preserve the duality of the bases (2.4) are given by automorphisms (2.5) where the matrix $C$ is either

$$
D_{1}=\left(\begin{array}{cccc}
0 & 0 & 1 & 0 \\
0 & 0 & -\frac{b_{2}}{b_{1}} & \frac{1}{b_{1}} \\
1 & b_{2} & -b_{2} b_{3} & b_{3} \\
0 & b_{1} & -b_{1} b_{3} & 0
\end{array}\right), \quad b_{1}, b_{2}, b_{3} \in \mathbb{R}, b_{1} \neq 0
$$

or

$$
D_{2}=\left(\begin{array}{cccc}
0 & 0 & -1 & 0 \\
0 & b_{1} & b_{1} b_{3} & 0 \\
-1 & b_{2} & b_{2} b_{3} & b_{3} \\
0 & 0 & \frac{b_{2}}{b_{1}} & \frac{1}{b_{1}}
\end{array}\right), \quad b_{1}, b_{2}, b_{3} \in \mathbb{R}, b_{1} \neq 0
$$

with determinants of $D_{1}$ and $D_{2}$ equal to \pm 1 . Note that $D_{1}$ is equal to the exchange matrix (3.9) for $b_{1}=1, b_{2}=b_{3}=0$. Inserting each of these matrices into (2.9)-(2.12) one gets again two different "dual" models.

The first model, obtained using (3.10) and parametrization $\tilde{g}=\mathrm{e}^{\tilde{g}_{1} \widetilde{T}^{1}} \mathrm{e}^{\tilde{g}_{2} \widetilde{T}^{2}}$, has flat background

$$
\widetilde{\mathcal{F}}\left(\tilde{g}_{1}, \tilde{g}_{2}\right)=\left(\begin{array}{cc}
0 & \frac{1}{b_{1}\left(b_{3}+\gamma\right)+\tilde{g}_{2}} \\
\frac{1}{b_{1}\left(\beta-b_{3}\right)-\tilde{g}_{2}} & \frac{\alpha+b_{2}(\beta+\gamma)}{\left(b_{1}\left(b_{3}-\beta\right)+\tilde{g}_{2}\right)\left(b_{1}\left(b_{3}+\gamma\right)+\tilde{g}_{2}\right)}
\end{array}\right),
$$

while background obtained using (3.11) is given by tensor

$$
\widetilde{\mathcal{F}}\left(\tilde{g}_{1}, \tilde{g}_{2}\right)=\left(\begin{array}{cc}
\frac{1}{\alpha-(\beta+\gamma)\left(b_{2}+b_{1} \tilde{g}_{2}\right)} & \frac{b_{1}\left(b_{3}+\beta\right)}{(\beta+\gamma)\left(b_{2}+b_{1} \tilde{g}_{2}\right)-\alpha} \\
\frac{b_{1}\left(b_{3}-\gamma\right)}{(\beta+\gamma)\left(b_{2}+b_{1} \tilde{g}_{2}\right)-\alpha} & -\frac{b_{1}^{2}\left(b_{3}+\beta\right)\left(b_{3}-\gamma\right)}{(\beta+\gamma)\left(b_{2}+b_{1} \tilde{g}_{2}\right)-\alpha}
\end{array}\right)
$$

with nonzero scalar curvature

$$
\widetilde{R}=\frac{4}{(\beta+\gamma)\left(b_{1} \tilde{g}_{2}+b_{2}\right)-\alpha} .
$$

One can see that once more we get two different Poisson-Lie T-dual sigma models no matter what the parameters $b_{1}, b_{2}$ and $b_{3}$ are.

It is well known that (non-)Abelian T-duality is induced by matrix $D_{0}$ that is a special case of $D_{1}$. In fact, dualizing $\mathcal{F}$ using $D_{0}$ we get tensor

$$
\widetilde{\mathcal{F}}^{\prime}\left(\tilde{g}_{1}, \tilde{g}_{2}\right)=\left(\begin{array}{cc}
0 & \frac{1}{\gamma+\tilde{g}_{2}} \\
\frac{1}{\beta-\tilde{g}_{2}}-\frac{\alpha}{\left(\beta-\tilde{g}_{2}\right)\left(\gamma+\tilde{g}_{2}\right)}
\end{array}\right)
$$


that can be brought to the form (3.12) by coordinate transformation

$$
\tilde{g}_{1}=\tilde{g}_{1}^{\prime}-\frac{b_{2} \tilde{g}_{2}^{\prime}}{b_{1}}, \quad \tilde{g}_{2}=\frac{\tilde{g}_{2}^{\prime}}{b_{1}}+b_{3} .
$$

Alternatively, it can be obtained by gauging the (non-)Abelian isometry and introduction of Abelian Lagrange multipliers. We shall investigate whether the duality induced by matrix $D_{2}$ can be obtained in a similar way.

Matrix $D_{2}$ can be transformed by automorpisms (2.14), (2.16) to the form

$$
D_{2}^{\prime}=\left(\begin{array}{cccc}
0 & 0 & -1 & 0 \\
0 & 1 & 0 & 0 \\
-1 & 0 & 0 & 0 \\
0 & 0 & 0 & 1
\end{array}\right) \text {. }
$$

Up to the change of sign necessary for being an automorphism of semi-Abelian Manin triple, matrix $D_{2}^{\prime}$ acts by switching $T_{1} \leftrightarrow \widetilde{T}_{1}$. One may suspect that this can actually be Buscher duality with respect to one-dimensional subgroup ${ }^{4}$ of isometry group $\mathscr{G}$. This subgroup is generated by left-invariant vector field $V_{1}=\partial_{g_{1}}-g_{2} \partial_{g_{2}}$ that together with $V_{2}=\partial_{g_{2}}$ satisfies (3.1).

To check our suspicion we have to find adapted coordinates $\left\{s_{1}, h_{1}\right\}$ such that $V_{1}$ becomes $V_{1}^{\prime}=\partial_{h_{1}}$ and $\mathcal{F}$ becomes independent of $h_{1}$. Suitable transformation of coordinates is given by

$$
g_{1}=h_{1}, \quad g_{2}=s_{1} \mathrm{e}^{-h_{1}} .
$$

Tensor (3.2) is then transformed to the form

$$
\mathcal{F}^{\prime}\left(s_{1}, h_{1}\right)=\left(\begin{array}{cc}
0 & \gamma \\
\beta & \alpha-(\beta+\gamma) s_{1}
\end{array}\right) .
$$

Treating $s_{1}$ as spectator we may dualize $\mathcal{F}^{\prime}$ with respect to $h_{1}$. Buscher rules that follow from (2.9)-(2.12) give tensor

$$
\tilde{\mathcal{F}}\left(s_{1}, \tilde{h}_{1}\right)=\left(\begin{array}{ll}
\frac{\beta \gamma}{(\beta+\gamma) s_{1}-\alpha} & \frac{\gamma}{(\beta+\gamma) s_{1}-\alpha} \\
\frac{\beta}{\alpha-(\beta+\gamma) s_{1}} & \frac{1}{\alpha-(\beta+\gamma) s_{1}}
\end{array}\right)
$$

and subsequent change of coordinates

$$
s_{1}=b_{1} \tilde{g}_{2}+b_{2}, \quad h_{1}=b_{1} b_{3} \tilde{g}_{2}-\tilde{g}_{1}
$$

restores (3.13).

Alternative formulation of duality given by matrix (3.15) follows from gauge invariant parent action

$$
\begin{aligned}
S\left[h_{1}, s_{1}, A_{-}, A_{+}, \tilde{h}_{1}\right]= & \frac{1}{2 \pi} \int D_{-} h_{1}\left(\alpha-s_{1}(\beta+\gamma)\right) D_{+} h_{1}+ \\
& +D_{-} h_{1} \beta \partial_{+} s_{1}+\partial_{-} s_{1} \gamma D_{+} h_{1}+\tilde{h}_{1}\left(\partial_{-} A_{+}-\partial_{+} A_{-}\right)
\end{aligned}
$$

\footnotetext{
${ }^{4}$ Dualities of this form are called factorised in [25].
} 
where

$$
D_{ \pm} h_{1}=\partial_{ \pm} h_{1}+A_{ \pm} h_{1}
$$

Integrating out gauge fields $A_{+}$and $A_{-}$we obtain sigma model with background tensor (3.18) that can be brought to the form (3.13) by coordinate transformation.

One may also ask why Buscher duality with respect to $V_{2}$ is not included in (3.10) or (3.11). The reason is that change of bases

$$
T_{1}^{\prime}=T_{1}, \quad T_{2}^{\prime}= \pm \widetilde{T}_{2}, \quad \widetilde{T}_{1}^{\prime}=\widetilde{T}_{1}, \quad \widetilde{T}_{2}^{\prime}= \pm T_{2}
$$

is not an automorphism of the Manin triple given by $(3.1)$ and $\left[\widetilde{T}_{1}, \widetilde{T}_{2}\right]=0$.

\section{Sigma models with four-dimensional target space}

One can also ask if there are some "Poisson-Lie identities" preserving the structure of semi-Abelian Manin triple, i.e. Poisson-Lie T-pluralities generated by automorphisms of $\mathfrak{d}=\mathfrak{g} \oplus \tilde{\mathfrak{g}}$ that preserve both Lie brackets (3.3) and duality of the basis (2.4). The answer is positive, and the mappings can have two possible forms given by matrices

$$
I_{1}=\left(\begin{array}{cccc}
1 & b_{2} & -b_{2} b_{3} & b_{3} \\
0 & b_{1} & -b_{1} b_{3} & 0 \\
0 & 0 & 1 & 0 \\
0 & 0 & -\frac{b_{2}}{b_{1}} & \frac{1}{b_{1}}
\end{array}\right), \quad b_{1}, b_{2}, b_{3} \in \mathbb{R}, b_{1} \neq 0
$$

and

$$
I_{2}=\left(\begin{array}{cccc}
-1 & b_{2} & b_{2} b_{3} & b_{3} \\
0 & 0 & \frac{b_{2}}{b_{1}} & \frac{1}{b_{1}} \\
0 & 0 & -1 & 0 \\
0 & b_{1} & b_{1} b_{3} & 0
\end{array}\right) \quad b_{1}, b_{2}, b_{3} \in \mathbb{R}, b_{1} \neq 0
$$

Using (4.1) in atomic Poisson-Lie T-plurality transformation of the model given by (3.2) we get a sigma model in flat background. The example, however, is not particularly illuminating since the condition (2.13) is not satisfied for (4.2) and plural background cannot be calculated. For further investigation we focus on sigma models in four-dimensional target space and introduce spectator fields.

In the papers $[22,23]$ Poisson-Lie T-duality with spectators was used to study nonAbelian T-duals of sigma models in flat Minkowski space. Given the metric $\eta=\operatorname{diag}(-1,1$, $1,1)$ in coordinates $\{t, x, y, z\}$, we consider Killing vectors

$$
T_{1}:=K_{3}=z \partial_{t}+t \partial_{z}, \quad T_{2}:=L_{2}+K_{1}=x \partial_{t}+(t+z) \partial_{x}-x \partial_{z}
$$


satisfying $\left[T_{1}, T_{2}\right]=T_{2}$. These vectors generate a solvable two-dimensional group $\mathscr{G}$ of symmetries of the background $\eta$. The coordinates $\left\{s_{1}, s_{2}, g_{1}, g_{2}\right\}$ given by ${ }^{5}$

$$
\begin{aligned}
& t=\frac{1}{2} e^{-g_{1}} \sqrt{\left|s_{1}\right|}\left(\operatorname{sgn}\left(s_{1}\right)+e^{2 g_{1}}\left(g_{2}^{2}+1\right)\right), \\
& x=-e^{g_{1}} g_{2} \sqrt{\left|s_{1}\right|} \\
& y=s_{2}, \\
& z=-\frac{1}{2} e^{-g_{1}} \sqrt{\left|s_{1}\right|}\left(\operatorname{sgn}\left(s_{1}\right)+e^{2 g_{1}}\left(g_{2}^{2}-1\right)\right)
\end{aligned}
$$

are adapted to the action of $\mathscr{G}$. After the transformation of coordinates (4.4) the flat metric acquires the simple form

$$
\mathcal{F}\left(s_{1}, s_{2}, g_{1}, g_{2}\right)=\left(\begin{array}{cccc}
-\frac{1}{4 s_{1}} & 0 & 0 & 0 \\
0 & 1 & 0 & 0 \\
0 & 0 & s_{1} & 0 \\
0 & 0 & 0 & e^{2 g_{1}} s_{1}
\end{array}\right)
$$

from which one gets $E(s)$ by setting $g_{1}=0$. The group $\mathscr{G}$ can be embedded into semiAbelian Drinfel'd double with algebraic structure (3.3) allowing to find dual/plural backgrounds. ${ }^{6}$

Inserting matrices (4.1) and (4.2) into (2.9)-(2.12) one gets two different sigma models on $\mathscr{N} \times \mathscr{G}$ related to the original model in background (4.5) by Poisson-Lie T-plurality. The first, obtained using (4.1), is given by background

$$
\mathcal{F}\left(s_{1}, s_{2}, g_{1}, g_{2}\right)=\left(\begin{array}{cccc}
-\frac{1}{4 s_{1}} & 0 & 0 & 0 \\
0 & 1 & 0 & 0 \\
0 & 0 & \left(b_{2}^{2}+1\right) s_{1} & b_{1} e^{g_{1}}\left(b_{2} s_{1}-b_{3}\right) \\
0 & 0 & b_{1} e^{g_{1}}\left(b_{3}+b_{2} s_{1}\right) & b_{1}^{2} e^{2 g_{1}} s_{1}
\end{array}\right)
$$

that is flat and torsionless for any values ${ }^{7}$ of $b_{1}, b_{2}, b_{3}$. The second background, obtained using (4.2), is given by tensor

$$
\mathcal{F}\left(s_{1}, s_{2}, g_{1}, g_{2}\right)=\left(\begin{array}{cccc}
-\frac{1}{4 s_{1}} & 0 & 0 & 0 \\
0 & 1 & 0 & 0 \\
0 & 0 & \frac{b_{3}^{2}}{s_{1}}+s_{1} & \frac{e^{g_{1}}\left(b_{3}-b_{2} s_{1}\right)}{b_{1} s_{1}} \\
0 & 0 \frac{e^{g_{1}}\left(b_{3}+b_{2} s_{1}\right)}{b_{1} s_{1}} & \frac{e^{2 g_{1}}}{b_{1}^{2} s_{1}}
\end{array}\right) .
$$

\footnotetext{
${ }^{5}$ The action of $\mathscr{G}$ is not free and transitive for $t+z=0$, i.e. for $s_{1}=0$. We have to restrict our calculations to coordinate patches with $s_{1} \neq 0$.

${ }^{6}$ For further details concerning the process of finding the adapted coordinates see [22] where this particular case was denoted $S_{2,20}$.

${ }^{7}$ Note that (4.1) reduces to identity matrix for $b_{1}=1, b_{2}=b_{3}=0$. Consequently, (4.6) reduces to (4.5).
} 
Similarly to the previous case, the torsion vanishes. However, the symmetric part of (4.7), i.e. the metric, has vanishing scalar curvature but nontrivial Ricci tensor. Using transformation of coordinates

$$
\begin{array}{ll}
s_{1}=\frac{3 u^{4}}{b_{1}^{2}}+\frac{6 u^{2} z_{3}}{b_{1}}-2 u v+z_{3}^{2}, & s_{2}=z_{4}, \\
g_{1}=\frac{1}{2} \ln \left(\frac{3 u^{2}}{b_{1}^{2}}+\frac{6 z_{3}}{b_{1}}-\frac{2 v}{u}+\frac{z_{3}^{2}}{u^{2}}\right), & g_{2}=\frac{b_{1} b_{3}}{\sqrt{\frac{3 u^{2}}{b_{1}^{2}}+\frac{6 z_{3}}{b_{1}}+\frac{z_{3}^{2}-2 u v}{u^{2}}}}-b_{1} u z_{3}-u^{3}
\end{array}
$$

one can bring this background to a $p p$-wave in the Brinkmann form

$$
d s^{2}=2 \frac{z_{3}^{2}}{u^{2}} d u^{2}+2 d u d v+d z_{3}^{2}+d z_{4}^{2}
$$

identified in $[22,23]$ to be one of the gauged WZW models considered in $[29,30]$. We see that we again get two different sigma models, this time produced by Poisson-Lie Tpluralities that do not change the Manin triple.

\section{Poisson-Lie T-dualities and pluralities generated by Poisson-Lie iden- tities}

Comparing transformation matrices (3.10), (3.11) that generate Poisson-Lie T-dualities and (4.1), (4.2) that generate Poisson-Lie identities one may notice that they are related by canonical duality matrix (3.9) that exchanges generators $T_{a}$ and $\widetilde{T}^{a}$. Indeed, it is easy to check that

$$
D_{1}=D_{0} \cdot I_{1}, \quad D_{2}=D_{0} \cdot I_{2}
$$

That means that all Poisson-Lie T-dualities described in subsection 3.2 can be obtained by canonical non-Abelian T-duality of sigma models generated by Poisson-Lie identities of the initial model. Writing (2.5) as

$$
\left(\begin{array}{l}
\widetilde{T} \\
T
\end{array}\right)=D_{0} \cdot\left(\begin{array}{l}
T \\
\widetilde{T}
\end{array}\right)=D_{0} \cdot I \cdot\left(\begin{array}{l}
T \\
\widetilde{T}
\end{array}\right)
$$

we verify that this holds not only for the four-dimensional semi-Abelian Drinfel'd doubles, but for general Drinfel'd double $(\mathscr{G} \mid \widetilde{\mathscr{G}})$.

One can try to obtain similar relation for Poisson-Lie T-pluralities described in subsection 3.1. However, question then is what is the "canonical Poisson-Lie T-plurality". Motivated by equations (5.1) we can define it for Manin triples $(\mathfrak{d}, \mathfrak{g}, \tilde{\mathfrak{g}})$ and $(\mathfrak{d}, \hat{\mathfrak{g}}, \overline{\mathfrak{g}})$ with (3.3) and (3.4) by

$$
C_{0}=\left(\begin{array}{cccc}
-1 & 0 & 0 & 0 \\
0 & 0 & 0 & 1 \\
0 & 0 & -1 & 1 \\
1 & 1 & 0 & 0
\end{array}\right)
$$


i.e. by the matrix $C_{1}$ with $b_{1}=1, b_{2}=b_{3}=0$. Relabeling $b_{1} \rightarrow \frac{1}{b_{1}}, b_{2} \rightarrow-b_{2}, b_{3} \rightarrow-b_{3}$ in $I_{1}$ and $b_{2} \rightarrow-b_{2}, b_{3} \rightarrow-b_{3}$ in $I_{2}$ we then have

$$
C_{1}=C_{0} \cdot I_{1}, \quad C_{2}=C_{0} \cdot I_{2}
$$

and all Poisson-Lie T-pluralities from subsection 3.1 can be obtained by Poisson-Lie Tplurality of sigma models generated by (5.2) and Poisson-Lie identities of the initial model. Unfortunately, the choice (5.2) is by far not unique, and, as opposed to duality, we can hardly call it "canonical" Poisson-Lie T-plurality.

We have obtained similar results for Poisson-Lie T-pluralities of sigma models embedded in six-dimensional Drinfel'd doubles.

\section{Conclusion}

The examples presented in sections 3 and 4 prove that families of sigma models related by Poisson-Lie T-plurality may depend not only on the algebraic structure of Manin triple but also on the way how the given Manin triple is embedded in the Drinfel'd doubles.

This holds for the Poisson-Lie T-duality as well, so we may ask what should be considered as "true" T-duality. Is it only the procedure introduced in [1-3] that uses gauging of initial sigma model and is alternatively described in [8] as an exchange of dual bases $T_{a}$ and $\widetilde{T}^{a}$ of the isotropic subalgebras of Manin triple? Or can we admit any linear transformation of bases of the Drinfel'd double that give decompositions isomorphic to the Manin triple obtained by the exchange?

Possible answer to the question above follows from expressions (2.3) and (2.9) of both initial and dual/plural tensor. Namely, from these expressions we can see that tensors $\mathcal{F}$ and $\widehat{\mathcal{F}}$ depend both on algebraic structure of Manin triple and bilinear forms $E(s), \widehat{E}(s)$. Poisson-Lie identity does not change the initial Manin triple (only its embedding in the Drinfel'd doubles) but changes the bilinear form $E(s)$ according to the formula (2.12). Subsequent canonical duality or plurality then changes the Manin triple and produce PoissonLie T-dual/plural tensor. Moreover, it turns out that non-canonical dualities may be hidden canonical dualities with respect to subgroups of the initial group of isometries.

Open Access. This article is distributed under the terms of the Creative Commons Attribution License (CC-BY 4.0), which permits any use, distribution and reproduction in any medium, provided the original author(s) and source are credited.

\section{References}

[1] T.H. Buscher, A symmetry of the String Background Field Equations, Phys. Lett. B 194 (1987) 59 [INSPIRE].

[2] X.C. de la Ossa and F. Quevedo, Duality symmetries from non-Abelian isometries in string theory, Nucl. Phys. B 403 (1993) 377 [hep-th/9210021] [INSPIRE].

[3] M. Roček and E.P. Verlinde, Duality, quotients and currents, Nucl. Phys. B 373 (1992) 630 [hep-th/9110053] [INSPIRE]. 
[4] K. Sfetsos and D.C. Thompson, On non-abelian T-dual geometries with Ramond fluxes, Nucl. Phys. B 846 (2011) 21 [arXiv: 1012.1320] [inSPIRE].

[5] Y. Lozano, E. Ó Colgáin, K. Sfetsos and D.C. Thompson, Non-abelian T-duality, Ramond Fields and Coset Geometries, JHEP 06 (2011) 106 [arXiv:1104.5196] [INSPIRE].

[6] G. Itsios, Y. Lozano, J. Montero and C. Núñez, The AdS $S_{5}$ non-Abelian T-dual of Klebanov-Witten as a $\mathcal{N}=1$ linear quiver from M5-branes, JHEP 09 (2017) 038 [arXiv: 1705.09661] [INSPIRE].

[7] G. Itsios, H. Nastase, C. Núñez, K. Sfetsos and S. Zacarías, Penrose limits of Abelian and non-Abelian T-duals of $A d S_{5} \times S^{5}$ and their field theory duals, JHEP 01 (2018) 071 [arXiv: 1711.09911] [INSPIRE].

[8] C. Klimčík and P. Ševera, Dual non-Abelian duality and the Drinfeld double, Phys. Lett. B 351 (1995) 455 [hep-th/9502122] [INSPIRE].

[9] C. Klimčík, Poisson-Lie T duality, Nucl. Phys. Proc. Suppl. 46 (1996) 116 [hep-th/9509095] [INSPIRE].

[10] P. Bouwknegt, M. Bugden, C. Klimčík and K. Wright, Hidden isometry of "T-duality without isometry", JHEP 08 (2017) 116 [arXiv:1705.09254] [INSPIRE].

[11] R. Borsato and L. Wulff, Integrable Deformations of T-Dual $\sigma$ Models, Phys. Rev. Lett. 117 (2016) 251602 [arXiv: 1609. 09834] [INSPIRE].

[12] R. Borsato and L. Wulff, On non-abelian T-duality and deformations of supercoset string $\sigma$-models, JHEP 10 (2017) 024 [arXiv:1706.10169] [INSPIRE].

[13] B. Hoare and F.K. Seibold, Poisson-Lie duals of the $\eta$ deformed symmetric space $\sigma$-model, JHEP 11 (2017) 014 [arXiv:1709. 01448] [INSPIRE].

[14] R. Von Unge, Poisson Lie T plurality, JHEP 07 (2002) 014 [hep-th/0205245] [InSPIRE].

[15] L. Hlavatý and L. Šnobl, Poisson-Lie T-plurality of three-dimensional conformally invariant $\sigma$-models. II. Nondiagonal metrics and dilaton puzzle, JHEP 10 (2004) 045 [hep-th/0408126] [INSPIRE].

[16] X. Gomez, Classification of three-dimensional Lie bialgebras, J. Math. Phys. 41 (2000) 4939.

[17] L. Hlavatý and L. Šnobl, Classification of Poisson-Lie T-dual models with two-dimensional targets, Mod. Phys. Lett. A 17 (2002) 429 [hep-th/0110139] [INSPIRE].

[18] L. Šnobl and L. Hlavatý, Classification of 6-dimensional manin triples, Int. J. Mod. Phys. A 17 (2002) 4043 [math.QA/0202209] [InSPIRE].

[19] L. Hlavatý, I. Petr and V. Štěpán, Poisson-Lie T-plurality with spectators, J. Math. Phys. 50 (2009) 043504.

[20] L. Hlavatý and F. Petrásek, On uniqueness of T-duality with spectators, Int. J. Mod. Phys. A 31 (2016) 1650143 [arXiv:1606.02522] [INSPIRE].

[21] L. Hlavatý and I. Petr, Plane-parallel waves as duals of the flat background, Class. Quant. Grav. 32 (2015) 035005 [arXiv: 1406.0971] [INSPIRE].

[22] F. Petrásek, L. Hlavatý and I. Petr, Plane-parallel waves as duals of the flat background II: T-duality with spectators, Class. Quant. Grav. 34 (2017) 155003 [arXiv:1612.08015] [INSPIRE]. 
[23] L. Hlavatý, I. Petr and F. Petrásek, Plane-parallel waves as duals of the flat background III: T-duality with torsionless B-field, Class. Quant. Grav. 35 (2018) 075012 [arXiv: 1711.08688] [INSPIRE].

[24] J. Patera, R.T. Sharp, P. Winternitz and H. Zassenhaus, Subgroups of the Poincaré Group and their Invariants, J. Math. Phys. 17 (1976) 977 [INSPIRE].

[25] D. Lüst and D. Osten, Generalised fluxes, Yang-Baxter deformations and the $O(d, d)$ structure of non-abelian T-duality, JHEP 05 (2018) 165 [arXiv: 1803.03971] [INSPIRE].

[26] E. Álvarez, L. Álvarez-Gaumé and Y. Lozano, On nonAbelian duality, Nucl. Phys. B 424 (1994) 155 [hep-th/9403155v4] [INSPIRE].

[27] B. Hoare and A.A. Tseytlin, Homogeneous Yang-Baxter deformations as non-abelian duals of the AdS $S_{5} \sigma$-model, J. Phys. A 49 (2016) 494001 [arXiv: 1609.02550v3] [InSPIRE].

[28] M. Hong, Y. Kim and E. Ó Colgáin, On non-Abelian T-duality for non-semisimple groups, Eur. Phys. J. C 78 (2018) 1025 [arXiv:1801.09567] [InSPIRE].

[29] A.A. Tseytlin, Exact solutions of closed string theory, Class. Quant. Grav. 12 (1995) 2365 [hep-th/9505052] [INSPIRE].

[30] A.A. Tseytlin, Exact string solutions and duality, in proceedings of the 2nd Journee Cosmologique within the framework of the International School of Astrophysics, D. Chalonge, Paris, France, 2-4 June 1994, pp. 371-398 [hep-th/9407099] [INSPIRE]. 